\title{
Gestion des ressources naturelles dans le Pays de Tulle : impacts paysagers et histoire du châtaignier (Castanea sativa Mill.) de l'Antiquité à la Renaissance d'après la palynologie
}

\begin{abstract}
RÉSUMÉ
Cet article présente les résultats paléoenvironnementaux des premières analyses palynologiques et sédimentologiques menées dans le pays de Tulle (Mars, Gimel-les-Cascades, Corrèze, France). Elles se fondent sur le croisement de différents indicateurs biotiques (grains de pollen, microfossiles non-polliniques) et sédimentaires. Neuf datations radiocarbone ont été réalisées. Cette recherche décrit les dynamiques des paysages végétaux depuis l'Antiquité sous l'action de leurs facteurs de contrôle climatiques et anthropiques. Elle analyse en quoi ces changements paysagers traduisent des gestions diversifiées des ressources végétales dont la culture du châtaignier (Castanea sativa Mill.). Arbre emblématique en Limousin, le châtaignier constitue un marqueur paysager et territorial mais dont les connotations ont souvent été contradictoires et fluctuantes en 2000 ans d'histoire.
\end{abstract}

\section{MOTS CLÉS}

palynologie, micro-fossiles non polliniques, histoire des activités humaines, Castanea sativa Mill., Corrèze

\section{RESUMEN}

Este artículo presenta los resultados paleoambientales de los primeros análisis palinolójicos y sedimentológicos llevados a cabo en el area de Tulle (Mars, Gimel-les-Cascades, Corrèze, Francia). Se basan en la comparación de distintos indicadores bióticos (polen, microfósiles no polínicos) y sedimentarios y en la realización de 9 dataciones radiocarbónicas. Esta investigación establece datos inéditos sobre las dinámicas paisajísticas desde la Antigüedad y sus factores de control tanto climáticos como antrópicos. También analiza de que manera estos cambios paisajísticos pueden significar el desarrollo de sistemas de uso diversificados de los recursos vegetales como el cultivo del castaño (Castanea sativa Mill.), árbol emblemático en el Limousin, pero cuyas connotaciones como marcador paisajístico y territorial han sido a menudo contradictorias y fluctuantes en 2000 años de historia.

\section{Palabras claves}

palinología, microfósiles no polínicos, historia de los usos del suelo, Castanea sativa Mill., Corrèze 


\section{INTRODUCTION ET SITE D'ÉTUDE}

Le Limousin est une région française où la question de "l'archéologie des paysages ruraux" s'est posée dès les années 70 en étudiant de manière interdisciplinaire les signatures sédimentaires et les données archéologiques $^{1}$. L'analyse des transformations paysagères et des processus d'anthropisation a ensuite pris une plus grande dimension en se fondant sur l'étude pluri-indicateurs des remblaiements de fonds de vallon et de sols de cultures fossilisés ${ }^{2}$. Dans toutes ces recherches, une place de premier plan a été occupée par la palynologie $^{3}$ qui a décrit les premiers impacts des sociétés sur leur environnement végétal ${ }^{4}$. À l'instar de recherches menées dans d'autres secteurs de moyennes montagnes comme le Jura ${ }^{5}$, la recherche palynologique menée en Limousin a ensuite recouvert de plus larges ambitions en s'intéressant à l'évolution des activités agro-sylvo-pastorales ayant contribué à la mise en place de véritables "paysages culturels", héritages d'une longue co-évolution entre les sociétés et leur environnement ${ }^{6}$. Dans ce champ thématique, une importante disparité géographique existe entre la montagne

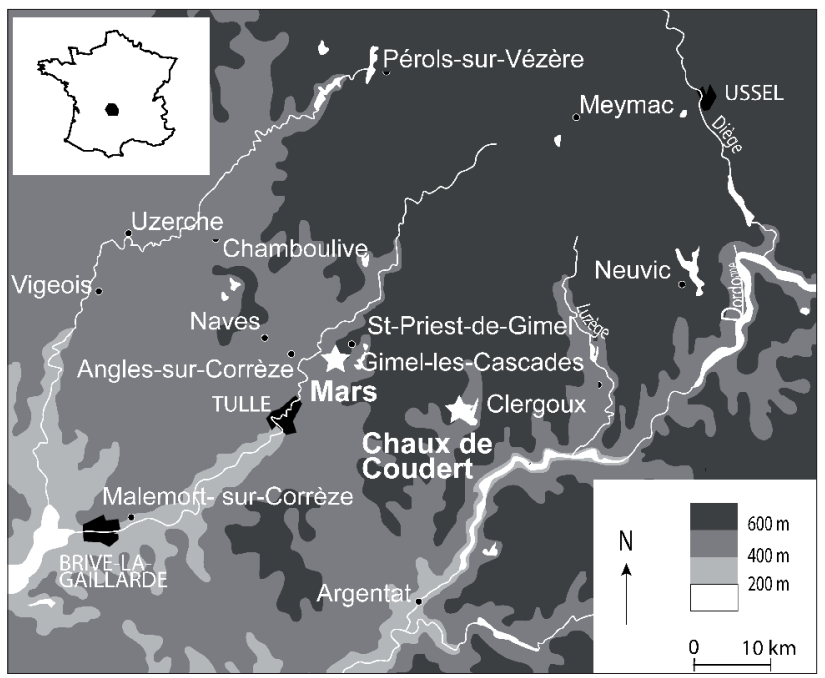

Fig. 1. Zone d'étude : situation des séquences de Mars (Gimel-les-Cascades) et de la Chaux de Coudert (Clergoux). limousine, plutôt bien étudiée ${ }^{7}$, et les plateaux périphériques, dont le pays de Tulle, où aucune étude palynologique n'avait encore été menée en dépit de la richesse du cadre archéologique ${ }^{8}$. Cet article présente les résultats paléoenvironnementaux des analyses palynologiques et sédimentologiques de la séquence de Mars (Gimel-les-Cascades, Corrèze), entreprises dans le cadre du programme Leader+ "Analyse spatiale $d u$ peuplement de la périphérie des sites de Tintignac (Naves, Corrèze) et du Mont Beuvray (Glux-enGlenne, Nièure)" (dir. J.-P. Guillaumet, UMR 6298) ${ }^{9}$. De par la qualité de l'enregistrement sédimentaire et l'analyse de bio-indicateurs complémentaires aux grains de pollen - les micro-fossiles non polliniques -, s'ancrant ainsi dans la droite ligne des travaux pionniers de M.-F. Diot ${ }^{10}$, cette recherche apporte des

\footnotetext{
Desbordes \& Valadas 1979.

Allée 2003.

Denèfle et al. 1980 ; Diot 1987.

Guenet 1992.

Gauthier 2004.

Berglund 2011.

Miras et al. 2010 ; 2011.

Maniquet 2008 ; Toledo i Mur et al. 2005.

9. Nous remercions G. Lintz (SRA Limousin), A. Bouchette (†) (Inrap), M. Cabanis (Inrap et GEOLAB, UMR 6042), R. Rouaud (GEOLAB, UMR 6042) et M. Brun (CR Limousin) pour leur aide dans la recherche bibliographique et leurs commentaires avisés. Nous remercions également P.-Y. Demars (UMR 5199) pour nous avoir fait découvrir le site des Chaux-de-Coudert. Nos plus vifs remerciements sont également adressés à H. Richard (UMR 6249), à l'origine de ce travail palynologique, et à J. Didier (UMR 6249) pour avoir pris en charge l'intégralité des préparations des échantillons. Ce projet a bénéficié d'un financement européen labellisé Leader+, associant la Communauté de Commune du Pays de Tulle et le Parc Naturel Régional du Morvan. Nous tenons à leur témoigner notre plus grande reconnaissance.

10. Diot \& Lopez-Saez 1998.
} 
données inédites sur les dynamiques paysagères depuis l'Antiquité. Elle analyse en quoi ces changements paysagers traduisent des exploitations du milieu par les sociétés sur près de 2000 ans d'histoire. Enfin, un accent tout particulier a été porté sur la gestion d'une ressource végétale importante et emblématique en Limousin : le châtaignier (Castanea sativa Mill.)

La région d'étude se situe dans le Limousin au cœur du département de la Corrèze (fig. 1). La tourbière soligène dite de Mars (45¹8’18,67 “N, 150’09,98”E, 430 m, commune de Gimel-les-Cascades) repose sur des leucogranites hétérogènes à loupes surmicacées et enclaves mobilisées de micaschistes à sillimanites ${ }^{11}$. Cette tourbière de petite superficie privilégie un enregistrement local de la végétation permettant une reconstruction à une échelle plus fine des interactions socio-environnementales ${ }^{12}$.

\section{MÉTHOdOLOGIE}

Deux types d'analyse ont été menés. L'analyse sédimentologique permet de caractériser l'environnement de dépôt des strates tourbeuses et d'identifier des arrivées détritiques au sein des strates tourbeuses. L'analyse palynologique (grains de pollen et spores de végétaux) a pour objectif de reconstituer l'évolution du couvert végétal et des activités agro-sylvo-pastorales menées dans le secteur d'étude. Le recours à des bio-indicateurs complémentaires que sont les microfossiles non polliniques (par exemple les spores d'algues ou les spores de champignons) permet de préciser ces restitutions paléoenvironnementales en dégageant les composantes locales. Ainsi, les spores de champignons coprophiles qui se développent sur les excréments d'herbivores peuvent attester la présence locale de bétail ${ }^{13}$.

\section{Carottage, prélèvement et datation radiocarbone}

Les carottages effectués en 2007 et 2008 ont été réalisés à l'aide d'un carottier russe (sonde GIK 50 cm, $5 \mathrm{~cm}$ de diamètre). Les échantillons ont été prélevés tous les $4 \mathrm{~cm}$ sur la totalité de la séquence de 1,88 $\mathrm{m}$ de puissance.

Neuf datations radiocarbone AMS ont été réalisées sur des échantillons de tourbe par le Poznan Radiocarbon Laboratory. Les âges calibrés (cal. BP, cal. a.C. et cal. p.C.) ont été obtenus à partir du programme CALIB REV 5.0.1. ${ }^{14}$. Ces dates sont présentées et critiquées dans la figure 2.

\begin{tabular}{|c|c|c|c|c|}
\hline Réf. Labo. & Prof. $(\mathrm{cm})$ & Date BP & Date cal. BP & Date cal. BC/AD \\
\hline Poz-26198 & 19 & \multicolumn{2}{|c|}{$\begin{array}{c}103.02 \pm 0.33 \mathrm{pMC} \\
\text { (sub-récent) }\end{array}$} & c. 1952 \\
\hline Poz-21166 & 31 & $440 \pm 30$ & $533-461$ & $1417-1489$ \\
\hline Poz-26199 & 42 & $495 \pm 30$ & $549-501$ & $1401-1449$ \\
\hline Poz-26200 & 60 & $430 \pm 30$ & $529-451$ & $1421-1499$ \\
\hline Poz-26201 & 82 & $635 \pm 30$ & $665-553$ & $1285-1397$ \\
\hline Poz-26202 & 100 & $775 \pm 30$ & $734-670$ & $1216-1280$ \\
\hline Poz-26223 & 140 & $990 \pm 35$ & $963-795$ & $987-1155$ \\
\hline Poz-21168 & 151 & $1105 \pm 30$ & $1065-952$ & $885-998$ \\
\hline Poz-21169 & 174 & $1640 \pm 30$ & $1613-1416$ & $337-534$ \\
\hline
\end{tabular}

Fig. 2. Les datations radiocarbone (calibration à $2 \square$ d'après Stuiver \& Reimer 2005).

11. Emberger \& Périchaud 1979.

12. Jacobson \& Bradshaw 1981.

13. Ejarque et al. 2011 ; Jouffroy-Bapicot 2010.

14- Stuiver \& Reimer 2005. 


\section{Analyses palynologiques et sédimentologiques}

L'extraction pollinique a été réalisée selon une méthode décrite par Faegri \& Iversen ${ }^{15}$. La somme pollinique moyenne comptée est de 400 grains de pollen de plantes vasculaires terrestres. L'identification taxonomique a été menée en utilisant différents atlas ${ }^{16}$ et les diagrammes sont construits à partir de Gpal Win ${ }^{17}$. Les fréquences polliniques ont été calculées sur la base des sommes polliniques totales, dont sont exclus les spores, les taxons aquatiques et hygrophytiques. Les pourcentages des microfossiles non polliniques ont été calculés sur la même somme de base. Les Indicateurs Polliniques d'Anthropisation (IPA) regroupant les anthropochores (principalement les céréales) et les apophytes (essentiellement les marqueurs pastoraux et rudéraux) sont ceux identifiés par la mise en place de référentiels polliniques actuels sur la Montagne limousine ${ }^{18}$.

Après une description de la lithologie des faciès de chaque carotte, différentes grandeurs physiques ont été mesurées suivant des protocoles standards ${ }^{19}$ à une résolution de $5 \mathrm{~mm}$. Les diagraphies Gamma Ray et acoustique ainsi que la susceptibilité magnétique permettent d'analyser la tourbe (densité, compaction, composition, teneur en eau) et de mettre en évidence des apports d'éléments détritiques.

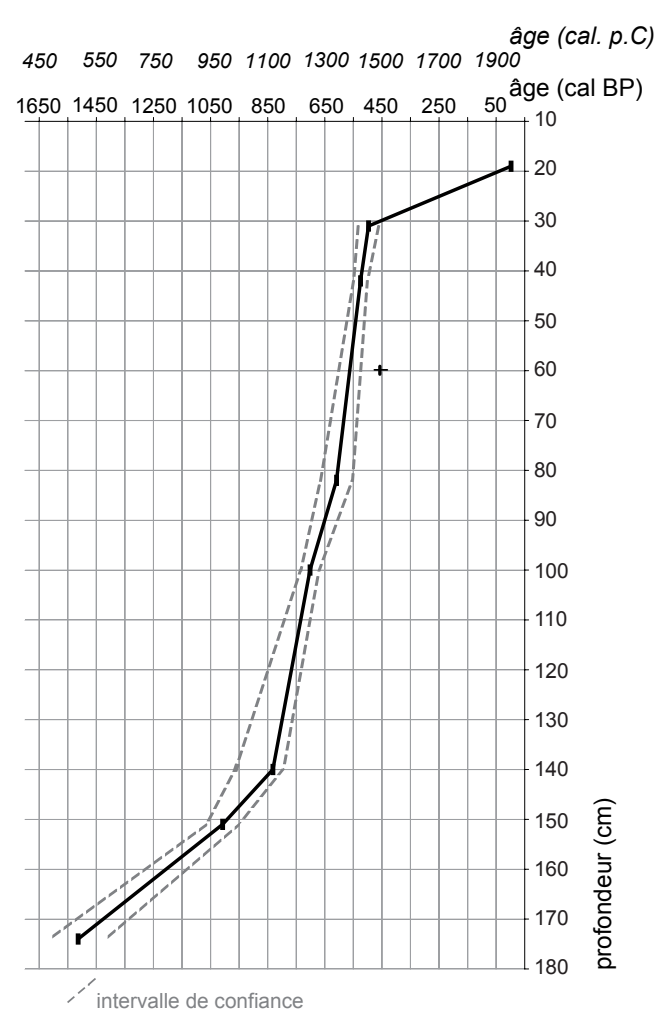

Fig. 3. Modèle âge-profondeur de la séquence de Mars.

\section{RÉSULTATS}

\section{Datations radiocarbone}

Le modèle âge-profondeur (fig. 3) a été établi à partir de l'interpolation linéaire de huit datations. La date Poz-26200 a été exclue en raison de l'inversion constatée entre elle et la date Poz-26199 qui est en cohérence totale avec les dates suivantes. L'enregistrement sédimentaire de Mars couvre un peu moins de deux millénaires, la base de la séquence étudiée remontant au Bas-Empire. Les taux de sédimentation assurent une bonne résolution d'analyse jusqu'au XVe $\mathrm{x}$. approximativement (de la base à $30 \mathrm{~cm}$ ). Par la suite, une brutale et importante diminution du taux de sédimentation ne permet plus de détailler aussi finement les restitutions paléoenvironnementales pour les périodes comprises entre le $\mathrm{XVI}^{\mathrm{e}} \mathrm{s}$. approximativement et la période sub-actuelle.

15- Faegri \& Iversen 1989.

16- Reille 1995-1999 ; van Geel 1978.

17. Goeury 1997.

18. Miras 2009.

19. Serra $1979 ; 1985$. 


\section{Analyse palynologique (fig. 4, 5 et 6)}

La zone pollinique M-1 se caractérise par une baisse progressive du rapport PA/T (Pollen d'Arbre sur le Total de grains de pollen comptés) permettant la distinction de deux sous-zones. Des fréquences polliniques élevées d'Alnus ${ }^{20}$ (c. $50 \%$ ) expliquent un rapport PA/T élevé à $70 \%$ (M-1a). Les autres essences arborées présentent des valeurs plus réduites : Quercus (10\%), Fagus et Betula (5\%). Castanea sativa et Juglans sont présents. La présence d'Abies est négligeable pour toute la séquence. Les Poaceae sont les principales composantes de l'assemblage pollinique herbacé (c. $20 \%$ ). Concernant les indicateurs polliniques d'anthropisation (IPA), des occurrences régulières de Cerealia-type et de Secale sont observées en même temps qu'une présence remarquable de différentes apophytes comme les types Rumex (2\%), Plantago, Plantago

Mars (Gime1-1es-Cascades, Corrèze, 430 m)

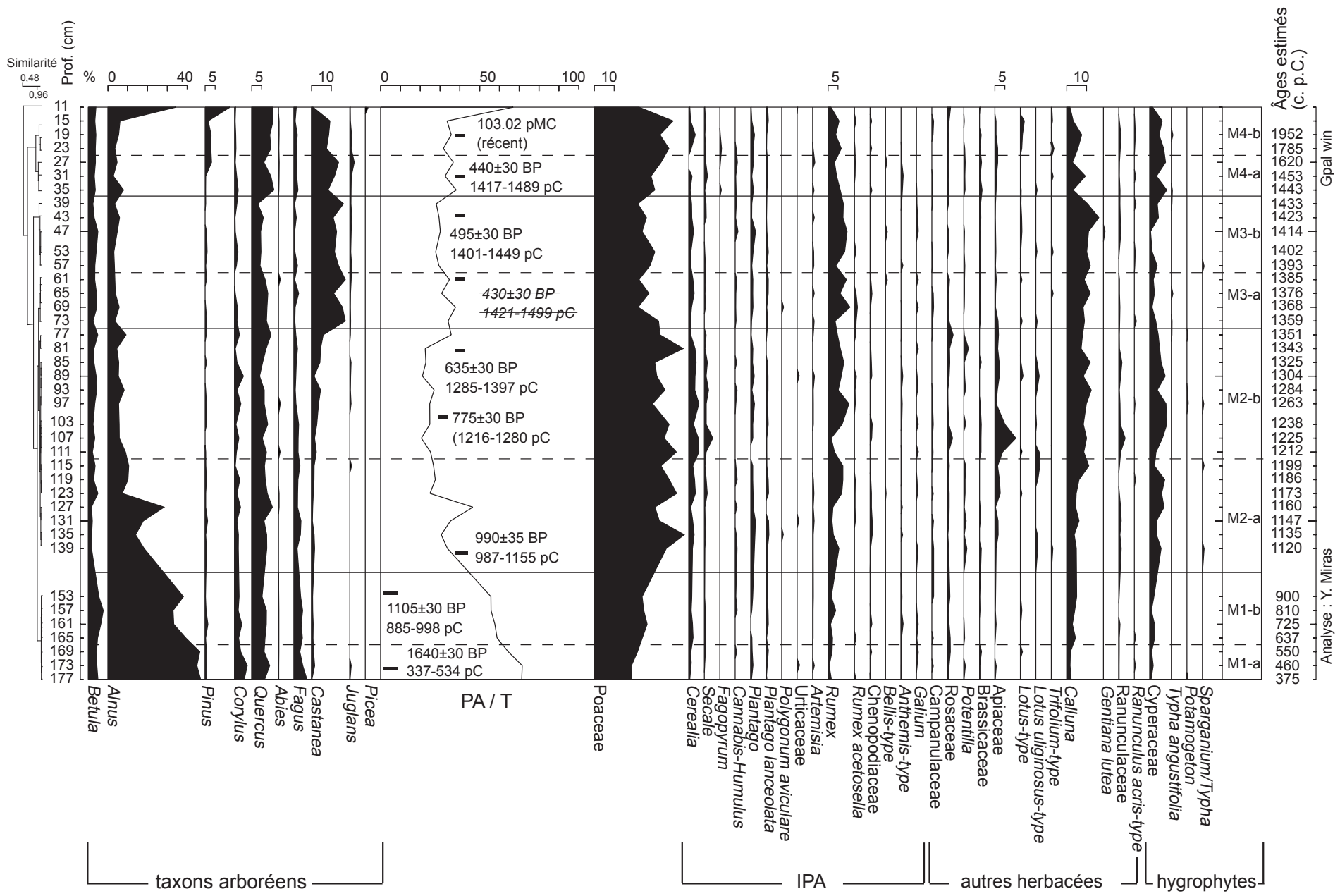

Fig. 4. Diagramme pollinique simplifié en fréquences relatives de la séquence de Mars.

20- La figure 6 récapitule les noms de famille, de genre et d'espèce présentés en latin dans le texte avec leur nom vernaculaire. 
Mars (Gime1-1es-Cascades, Corrèze, $430 \mathrm{~m}$ )

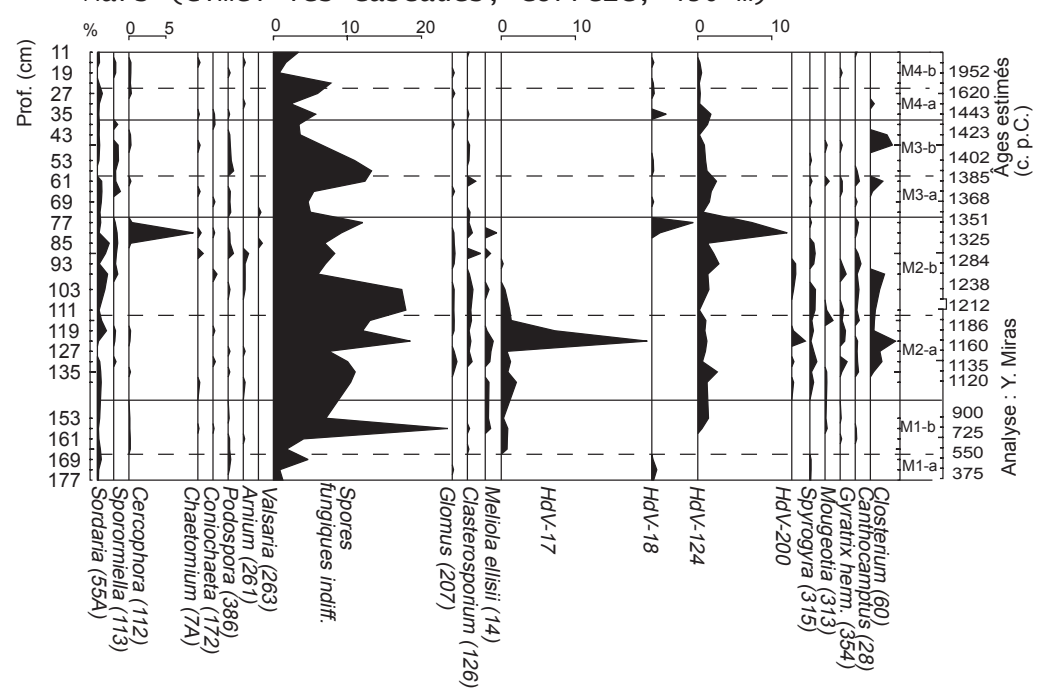

Fig. 5. Diagramme simplifié de micro-fossiles non polliniques en fréquences relatives de la séquence de Mars.

\begin{tabular}{|c|c|c|c|}
\hline FAMILLE, NOMS DE GENRE OU D'ESPĖCE & NOM VERNACULAIRE & FAMILLE, NOMS DE GENRE OU D'ESPĖCE & NOM VERNACULAIRE \\
\hline Abies & sapin & Lotus uliginosus & lotier des marais \\
\hline Alnus & aulne & Picea & épicéa \\
\hline Apiaceae & Apiacées & Pinus & pin \\
\hline Artemisia & armoise & Plantago & plantain \\
\hline Anthemis & anthémis & Plantago lanceolata & plantain lancéolé \\
\hline Bellis & pâquerette & Poaceae & Poacées \\
\hline Betula & bouleau & Polygonum aviculare & renouée des oiseaux \\
\hline Campanulaceae & Campanulacées & Potamogeton & potamot \\
\hline Calluna & callune & Potentilla & potentille \\
\hline Cannabis-Humulus & chanvre-houblon & Quercus & chêne \\
\hline Castanea & châtaignier & Ranunculaceae & Renonculaceae \\
\hline Cerealia & céréale & Ranunculus acris & renoncule âcre \\
\hline Chenopodiaceae & Chénopodiacées & Rosaceae & Rosacées \\
\hline Corylus & noisetier & Rumex & oseille \\
\hline Cyperaceae & Cypéracées & Rumex acetosella & petite oseille \\
\hline Fagus & Hêtre & Secale & seigle \\
\hline Fagopyrum & sarrasin & Sedum & orpin \\
\hline Galium & gaillet & Sparganium & rubanier \\
\hline Gentiana lutea & Gentiane jaune & Trifolium & trèfle \\
\hline Hypericum & millepertuis & Typha & massette \\
\hline Juglans & noyer & Urticacées & Urticacées \\
\hline
\end{tabular}

Fig. 6. Inventaire des taxons cités en latin dans le texte et leur nom vernaculaire. 
lanceolata ou Galium. Des plantes nitrophiles sont notées comme les Urticaceaes ou le type Rumex acetosella. Localement, les Cyperaceae présentent des valeurs de $2 \%$. Des occurrences régulières de Sordaria (HdV$55 \mathrm{~A})^{21}$ et de Podospora (HdV-386) sont contemporaines de notations du type HdV-18 et de Spirogyra (HdV315). Le passage à la sous-zone M-1b est marqué par la forte baisse du rapport PA/T expliquée par un recul des valeurs d'Alnus (30\%) et de tous les arbres à l'exception de Betula (10\%). Les fréquences des Poaceae et de Calluna augmentent respectivement à 30 et $5 \%$. La courbe continue de Cerealia-type démarre en même temps que s'étoffe le cortège de marqueurs anthropiques (Rumex : $4 \%$ et Anthemis). Une diversification des spores coprophiles se manifeste avec les notations simultanées de Sordaria (HdV-55A), de Cercophora (HdV112), Podospora (HdV-386), Chaetomium (HdV-7A), Coniochaeta cf. ligniaria (HdV-172) et Arnium (HdV-261). Les fréquences des spores fongiques indifférenciées progressent fortement à $25 \%$. Des observations de Gyratrix hermaphroditus (HdV-354) sont faites en même temps que démarre la courbe continue du type HdV124.

L'accentuation du déclin du rapport PA/T définit la zone pollinique M-2. Les pourcentages d'Alnus (de 20 à $10 \%$ approximativement) et de Fagus (de 4 à $1 \%$ ) baissent. En fonction de l'évolution des fréquences du châtaignier et des IPA, deux sous-zones ont été créées. Les Poaceae et Calluna présentent des valeurs accrues (respectivement 40 et $7 \%$ ) (M-2a) tout comme les anthropochores : Cerealia-type (4\%), Castanea sativa (2\%), courbes continues de Secale et du type Cannabis-Humulus. La représentation des autres indicateurs anthropiques s'intensifie, se pérennise et se diversifie : Plantago et Plantago lanceolata (2\%), fréquences maximales jusqu'à $10 \%$ du type Rumex, Artemisia, Chenopodiaceae. La strate herbacée s'enrichit nettement en taxons héliophiles (Campanulaceae, Renonculaceae, Rosaceae). Localement les Cyperaceae s'étendent (10 \%) de même que les Apiaceae. L'assemblage des spores de champignons coprophiles s'enrichit de Sporormiella (HdV-113). D'autres spores fongiques sont notées : Glomus (HdV-207), Clasterosporium caricinum (HdV-126), Meliola ellisii (HdV-14), HdV-200. Le type HdV-17 présente des valeurs maximales de $20 \%$. Les spores d'algues sont plus fréquentes (Spirogyra (HdV-315) de même que les Turbellariées (Gyratrix hermaphroditus) et Closterium idiosporum (HdV-60). Toutes ces tendances s'accélèrent dans la sous-zone M-2b avec un ratio PA/T minimal autour de $20 \%$. Lassemblage pollinique est largement dominé par les taxons de la strate herbacée : Poaceae (variations entre 30 et $40 \%$ ), Calluna (13\%), Cerealia-type (5\%). Les plantes commensales de culture (Anthemis) ou les marqueurs agro-pastoraux présentent une hausse remarquable. Localement, les taux des Apiaceae progressent (maximum à $11 \%$ ) et quelques plantes aquatiques sont notées sporadiquement (Potamogeton). Les spores fongiques dominent, notamment les spores coprophiles, essentiellement Sordaria (HdV-55a : 2 \%), Sporormiella (HdV-113), Cercophora (HdV-112) et Arnium (HdV. 261).

La stabilité du rapport $\mathrm{PA} / \mathrm{T}$ à des valeurs basses autour de $30 \%$ marque la zone pollinique $\mathrm{M}-3$. Les fluctuations enregistrées s'expliquent principalement par les variations des pourcentages de Castanea, justifiant la création de deux sous-zones. Alors que tous les arbres enregistrent des valeurs inférieures à 10 $\%$, Castanea sativa présente un maximum stable à des taux avoisinant les $20 \%$ (M-3a). Les fréquences des Poaceae et de Calluna ne varient guère et seuls les indicateurs polliniques d'agriculture semble marquer sensiblement le pas (Cerealia-type : $2 \%$ ). Les occurrences du type Cannabis-Humulus sont très serrées et les plantes rudérales se maintiennent très bien (principalement Plantago et Plantago lanceolata). Localement, les Cyperaceae et les Apiaceae régressent. L'assemblage des spores fongiques coprophiles est globalement stable et seuls les reculs de Glomus (HdV-207) et du type HdV-124 méritent d'être soulignés tout comme la rétraction des spores d'algues. L'inverse est ensuite constaté (M-3b). Les fréquences de Castanea sativa régressent à $10 \%$ tandis que progressent les taux de Juglans ( $2 \%$ ) et qu'un net regain des différentes anthropochores et

\footnotetext{
21- HdV pour Hugo-de-Vries Laboratory, université d'Amsterdam.
} 
apophytes apparaît. Parallèlement, les Poaceae recouvrent des fréquences supérieures à $30 \%$ tandis que celles de Calluna sont maximales à $16 \%$. Une diversification du tapis herbacé se manifeste par les notations conjointes des types Bellis, Trifolium, Lotus, Gentiana lutea, Ranunculus acris. Les spores de champignons coprophiles dominent avec principalement Sporormiella (HdV-113) et Podospora (HdV-386).

La dernière zone pollinique M-4 se définit par un retour à la hausse du rapport PA/T à près de $40 \%$ essentiellement expliquée par un regain des fréquences de Quercus à $12 \%$ et une réapparition modeste de Fagus. Les Poaceae restent très largement représentées (40\%). Toujours à partir des fréquences du châtaignier et des IPA, deux sous-zones ont été individualisées. Un premier recul des fréquences de Castanea sativa est corrélé à des représentations des types Cerealia et Secale en léger déclin (M-4a). Fagopyrum fait son apparition. Hormis la nette baisse des taux du type Rumex (à $5 \%$ ) et un arrêt des notations de Galium, le cortège de plantes rudérales et ségétales est toujours assez bien représenté. Une légère inflexion semble être mise au jour parmi les spores coprophiles dominées par Sordaria (HdV-55A). Dans la dernière sous-zone M-4b, les fréquences de Castanea sativa passent sous la barre des $10 \%$ alors que d'autres essences arborées voient leur expression augmenter de manière significative : Pinus (de 4 à $15 \%$ ) et Alnus (augmentation à $35 \%$ ). Les taxons herbacés et les IPA se maintiennent assez bien au moins pour la première moitié de la zone puis déclinent fortement comme les spores fongiques coprophiles. Le rapport PA/T retrouve des fréquences très élevées (65\%) en même temps que des grains de pollen de Picea apparaissent.

\section{Analyse sédimentologique}

Neuf unités stratigraphiques ont été définies à partir des variations des grandeurs physiques mesurées.

Unité I : la carotte est composée de la roche mère dégradée. L'allure décroissante des courbes de densité et de susceptibilité magnétique indique que cette dégradation est plus prononcée du bas vers le haut.

Unité II : la courbe de densité montre une tendance à la baisse jusqu'à la cote $152 \mathrm{~cm}$ (minimum de $\left.0,4 \mathrm{~g}_{\mathrm{gm}} \mathrm{cm}^{-3}\right)$ tandis que la courbe de susceptibilité magnétique est en hausse. Cette couche devient plus organo-argileuse de bas en haut. À partir de $152 \mathrm{~cm}$, un épisode sableux fait décroittre la susceptibilité magnétique et provoque un pic de densité et un bouleversement des valeurs de vitesse des ondes $\mathrm{P}$.

Unité III : une tendance à la baisse des valeurs de densité et l'observation indiquent que le faciès devient plus organique.

Unité IV : le faciès redevient plus organo-argileux avec une hausse de la densité. La valeur générale de la susceptibilité magnétique est légèrement plus haute $\left(-4.10^{-5} \mathrm{SI}\right)$ que dans l'unité III $\left(-5.10^{-5} \mathrm{SI}\right)$.

Unité $\mathrm{V}$ : de faibles valeurs de susceptibilité magnétique $\left(-5.10^{-5} \mathrm{SI}\right)$ et une légère baisse de densité à $0,75 \mathrm{~g} \cdot \mathrm{m}^{-3}$ indiquent que la base de l'unité est très organique. Les grandeurs physiques recouvrent ensuite des valeurs identiques à celles de l'unité IV $\left(0,8 \mathrm{~g} \cdot \mathrm{m}^{-3}\right.$ et $\left.-4 \cdot 10^{-5} \mathrm{SI}\right)$ indiquant un faciès organo-argileux.

Unité VI : elle correspond à un niveau organo-argileux dans lequel est insérée une couche organosableuse. Si la présence de sable ne semble pas influencer la susceptibilité magnétique qui est forte $\left(-3,5 \cdot 10^{-5} \mathrm{SI}\right)$, elle explique une forte hausse de la densité à $1,25 \mathrm{~g} . \mathrm{cm}^{-3}$.

Unité VII : c'est une unité organo-argileuse, dont les valeurs relativement stables de la susceptibilité magnétique $\left(-4.10^{-5} \mathrm{SI}\right)$ sont inférieures à celles de l'unité VI. La densité moyenne est de $1 \mathrm{~g} . \mathrm{cm}^{-3}$.

Unite VIII : dans cette unité organo-argileuse, deux épisodes sableux sont à l'origine des taux supérieurs à $1 \mathrm{~g} . \mathrm{cm}^{-3}$ de densité tandis que les valeurs de susceptibilité magnétique sont similaires à celles de l'unité VI $\left(-3,5 \cdot 10^{-5} \mathrm{SI}\right)$. Cette unité se différencie par une vitesse des ondes $\mathrm{P}$ très instable (2000 et $\left.4500 \mathrm{~m} \cdot \mathrm{s}^{-1}\right)$.

Unite IX : la base de nature organo-argileuse présente des vitesses d'ondes P (3000 m.s $\left.\mathrm{s}^{-1}\right)$ et une

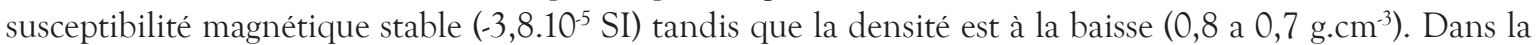
seconde partie, correspondant au sol tourbeux, la vitesse des ondes P est variable (entre 2000 et $7000 \mathrm{~m} . \mathrm{s}^{-1}$ ) indiquant une plus faible compaction. 


\section{DisCUSSION}

\section{Dynamiques paysagères et évolution des activités agro-sylvo-pastorales}

L'enregistrement sédimentaire organique démarre entre c. les $\mathrm{IV}^{\mathrm{e}}$ et $\mathrm{VI}^{\mathrm{e}} \mathrm{s}$. p.C. à la faveur d'une phase de calme morphogénique observée dans tout le Limousin et permettant le démarrage de la turfigenèse dans les lits fluviaux ${ }^{22}$. Une contribution anthropique peut également être avancée dans la mesure où des activités humaines, détectées dès la base (zone pollinique M1-a, fig. 4), ont pu bouleverser l'équilibre hydrique comme c'est le cas dans l'est du Massif central notamment pendant la période gallo-romaine ${ }^{23}$. Les forêts autour de Mars, essentiellement des chênaies et des chênaies-hêtraies, sont déjà dégradées et, dans un milieu régionalement bien ouvert, les pelouses occupent un grand espace. Les indices polliniques montrent l'existence d'activités agro-pastorales. Les marqueurs polliniques (anthropochores et plantes nitrophiles et de milieux piétinés) révèlent une certaine proximité des terres cultivées et pâturées même si la zone humide proprement dite ne semble pas encore fréquentée par le bétail. En effet, la tourbière de Mars est recouverte d'une aulnaie assez dense et les taux de spores fongiques coprophiles restent très modestes (fig. 5).

Une intensification de l'emprise humaine est manifeste à partir des $\mathrm{VI}^{\mathrm{e}}$ et $\mathrm{VII}^{\mathrm{e}} \mathrm{s}$. et elle concerne toutes les échelles spatiales (zone pollinique M1-b). Localement, le défrichement de l'aulnaie fait écho au recul des formations forestières plus régionales. L'accroissement et la pérennisation de tous les indicateurs polliniques d'anthropisation attestent, d'une part, cette intensification de la pression agro-pastorale à partir de l'Antiquité tardive et, d'autre part, la forte proximité des espaces pâturés et des terres cultivées. L'accroissement et la diversification des spores fongiques coprophiles (entre autres Sordaria, Cercophora et Podospora) témoignent d'une présence de bétail sur la tourbière si bien qu'un changement de la qualité des eaux est constaté grâce aux observations d'œufs de Turbellariées, indicateurs de conditions plus eutrophiques ${ }^{24}$. Cette plus forte attraction pour les zones humides, décrite dans d'autres secteurs du Limousin à une période similaire (la Boisserie, en Haute-Vienne ${ }^{25}$ ), peut s'expliquer par le fait que les exploitants pouvaient y trouver du fourrage, bien que de médiocre qualité. Il semble que ceci soit d'autant plus vrai que la zone humide est proche d'un habitat permanent ce qui semble être le cas à Mars même si les données archéologiques sont assez lacunaires. Des vestiges gallo-romains (substructions et tegulae) ont été repérés au village du Breuil, à la limite des communes de Gimel et de Saint-Priest-de-Gimel ${ }^{26}$ (fig. 1) ${ }^{27}$. De même, des tegulae, des briques et un flacon prismatique en verre, quoique de facture apparemment plus ancienne (c. $\mathrm{I}^{\mathrm{er}}-\mathrm{III} \mathrm{e}^{\mathrm{e}} \mathrm{s}$. p.C.), ont été mis au jour à la Cheynie, au lieu-dit de la Tuilière ${ }^{28}$. Enfin, la voie romaine Clermont-Périgueux pourrait passer par Lestrade et Lavergne ${ }^{29}$, soit à proximité immédiate du secteur d'étude.

Cette hausse de l'anthropisation repérée par l'analyse pollinique entre l'Antiquité et le Haut Moyen Âge se vérifie également pour la Montagne limousine particulièrement entre les VIII et IX s. p.C. ${ }^{30}$. Les sources écrites abondent dans ce sens. D'après B. Barrière ${ }^{31}$, “le Limousin d'avant l'an Mil n'était pas du tout ce no man's

\footnotetext{
2. Allée 2003, 255.

3. Cubizolle et al. 2011, 94.

4aas 1996, 371

- Allée et al. 1997, 373-375.

- Lintz 1992, 166.

27. Les données archéologiques citées procèdent d'opérations anciennes. Bien que situées dans un environnement proche de la zone

humide, elles ne peuvent malheureusement pas être localisées précisément.

28. Lintz 1992, 166.

29. Vazeille 1962, 175-176.

30. Miras et al. 2010, 109

31. Barrière 2006, 115.
} 
land forestier et broussailleux". Les sources carolingiennes, et notamment le cartulaire de Beaulieu (IX'-XII ${ }^{\mathrm{e}}$ s.) relatent l'existence de domaines parfaitement constitués avec des villae ${ }^{32}$. Cette phase semble même revêtir un caractère général à l'échelle de tout le Massif central. Des espaces agro-pastoraux sont gagnés sur les forêts aussi bien dans la Chaîne des Puys, où les aulnaies sont défrichées autour des $\mathrm{VI}^{\mathrm{e}}-\mathrm{VII}^{\mathrm{e}} \mathrm{s}^{33}{ }^{33}$, que dans l'Aubrac ou le Mont Lozère ${ }^{34}$ où les pâturages d'altitude commencent alors à s'étendre. Dans le Morvan, une diversification des pratiques avec le démarrage d'activités minières et/ou métallurgiques caractérise cette période ${ }^{35}$. Au vu de l'importance de cette phase à l'échelle macro-régionale, l'impact réel et souvent avancé des "invasions" ou plutôt des groupes migrants ${ }^{36}$ sur les occupations du sol durant cette période en Limousin peut être remis en question.

Les données sédimentologiques, à savoir une baisse brutale de la susceptibilité magnétique et une hausse de la densité, identifient une décharge détritique postérieurement au niveau $151 \mathrm{~cm}$ (fig. 7). Ce niveau de sable, compris entre 150 et $146 \mathrm{~cm}$ (Unité II) signe un épisode de crue ayant eu lieu postérieurement à 885 . 998 p.C. (Poz-21168). Cet important changement paléohydrologique enregistré dans la séquence tourbeuse de Mars est très probablement à relier avec l'instauration, entre les milieux du XI ${ }^{\mathrm{e}}$ et du XII ${ }^{\mathrm{e}}$ s., de nouvelles conditions climatiques plus froides correspondant à une baisse de l'activité solaire, et plus communément appelée l'“Oort Minimum" 37 . Il n'est malheureusement pas possible d'envisager également une contribution anthropique à cet événement paléohydrologique - des défrichements dans le bassin versant peuvent accroitre la disponibilité en sédiment - ni d'analyser les conséquences de cette variation climatique sur les sociétés dans la mesure où les niveaux correspondant se sont avérés pauvres en grains de pollen. Néanmoins, à partir de la seconde moitié du XII ${ }^{\mathrm{e}} \mathrm{s}$. jusqu'au milieu du XIV ${ }^{\mathrm{e}}$ s. approximativement (zone pollinique M-2), l'amplification des défrichements tant de l'aulnaie, qui régresse irrémédiablement sur la tourbière, que des formations forestières des environs donne naissance à un milieu localement et régionalement très ouvert. Dès le $\mathrm{XII}^{\mathrm{e}} \mathrm{s}$. dominent les formations herbacées pâturées et les données polliniques indiquent une activité pastorale d'intensité plus forte (zone pollinique M2-a). Le site de Mars est même au cœur d'une zone pastorale fréquentée par les troupeaux comme le démontrent les fréquences maximales des Poacées et les taux très élevés de marqueurs pastoraux locaux qu'ils s'agissent de marqueurs polliniques tels Potentilla ou Galium $^{38}$ ou des premières notations des spores de Sporormiella, champignon coprophile strict ${ }^{39}$. Les terres cultivées s'étendent comme l'indiquent les progressions des fréquences des céréales et des plantes commensales (Polygonum aviculare, Anthemis) telle Rumex dont la forte association avec la pratique agricole a été démontrée en Limousin ${ }^{40}$. Le Moyen Âge classique semble donc se caractériser par un schéma d'intensification et de diversification des pratiques territoriales. À une exploitation pastorale permanente dans un milieu ouvert, incluant les zones humides, s'ajoute une diversité des cultures avec une place accrue pour le seigle et le chanvre. On retrouve cette même tendance dans la gestion des ressources végétales dans l'ensemble du Massif central et notamment dans le Morvan ${ }^{41}$ ou la Chaîne des Puys où le XII s. voit les premiers indices polliniques de rouissage du chanvre dans les eaux du lac d'Aydat ${ }^{42}$.

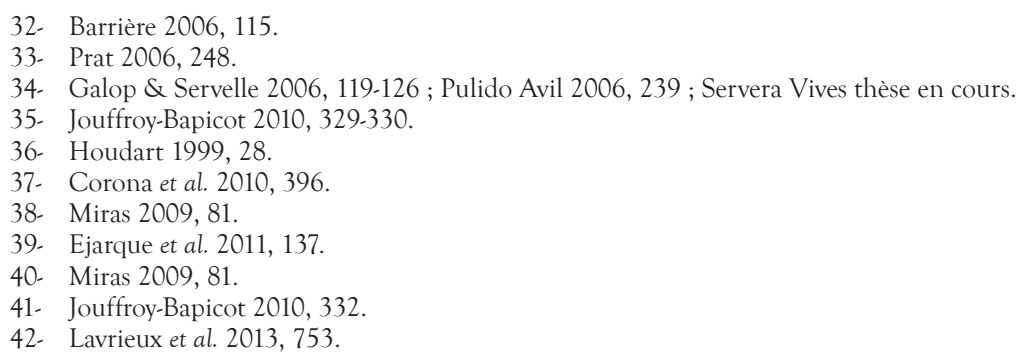


Mars (Gime1-1es-Cascades, Corrèze, 430 m)

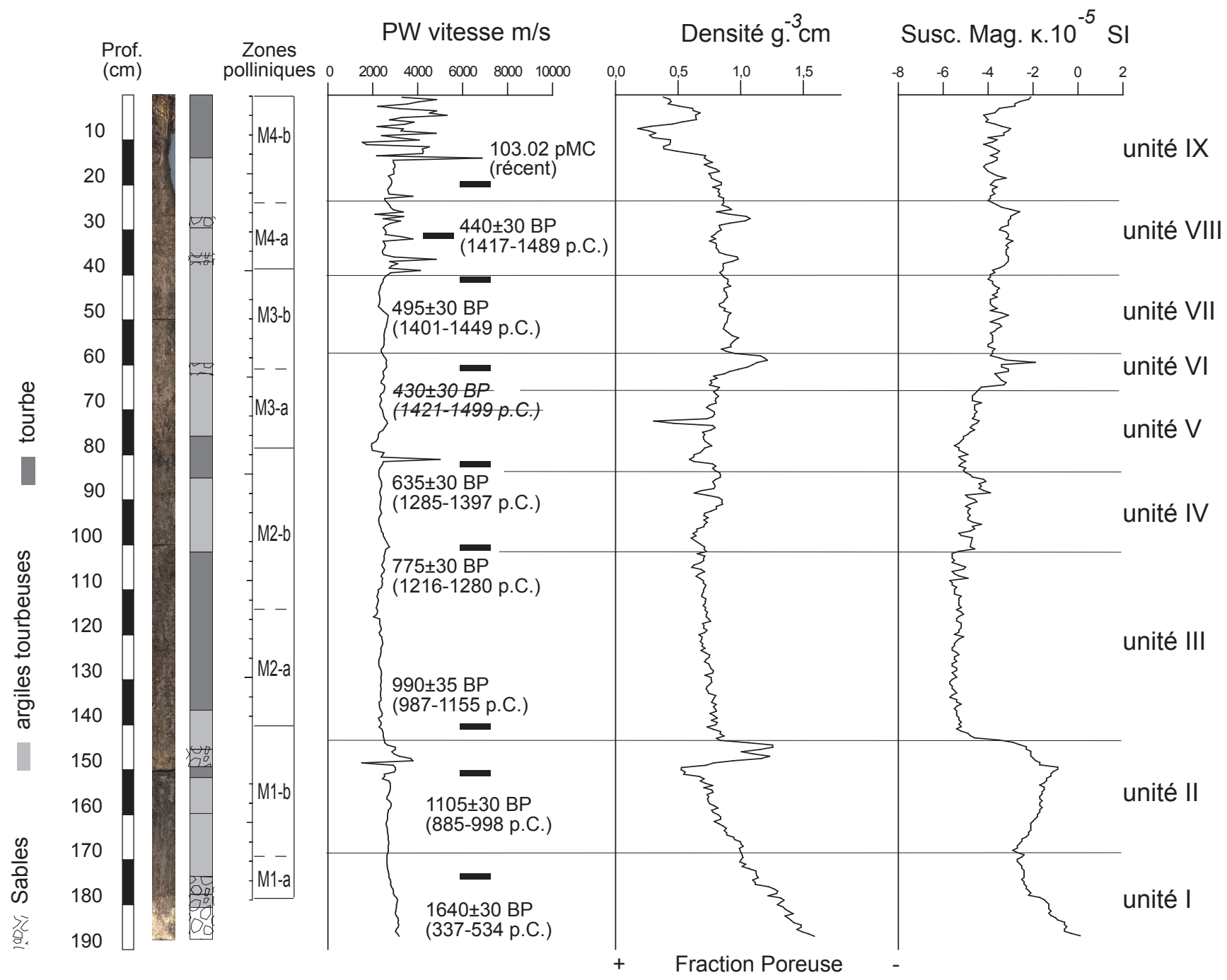

Fig. 7. Analyses lithostratigraphiques et sédimentologiques de la séquence de Mars. 
Cette forte pression anthropique responsable d'une extension manifeste des territoires agro-pastoraux s'accentue au cours du XIII ${ }^{\mathrm{e}}$ s. et jusqu'à la première moitié du XIV ${ }^{\mathrm{e}} \mathrm{s}$. (zone pollinique M-2b). Les fréquences polliniques minimales des principaux arbres attestent un milieu très ouvert laissant la part belle à un nouvel essor des emblavements, en seigle particulièrement. L'intensité des activités pastorales est plus forte et la zone humide de Mars évolue progressivement vers un pré humide pâturé comme le démontrent les enregistrements polliniques de Lotus uliginosus-t et des Apiaceae concomitantes d'un essor remarquable des spores fongiques coprophiles (Sporormiella). Cette période correspond de surcroît au Petit Optimum Médiéval, période située entre la seconde moitié du XII ${ }^{\mathrm{e}} \mathrm{s}$. et le XIII ${ }^{\mathrm{e}} \mathrm{s}$., qui a bénéficié d'étés particulièrement chauds et secs et d'hivers moins rigoureux ${ }^{43}$. La zone humide de Mars présente des faciès plus secs comme l'indique la hausse de Calluna et l'apparition de microfossiles non-polliniques révélateurs d'un engorgement moins prononcé (HdV-200) et non permanent (HdV-123 et HdV-28). Le contexte bioclimatique et les besoins en espace toujours plus impérieux sont tels que les zones humides font désormais totalement partie du système d'exploitation et sont gérées dans ce sens.

Cette phase de forte emprise semble marquer légèrement le pas dans le pays de Tulle durant la seconde moitié du XIV $\mathrm{s}$. (fin de la zone pollinique M2-b et zone pollinique M3-a). Les essors conjugués des fréquences polliniques de Quercus et de Fagus révèlent un regain modéré des forêts. Localement, les données palynologiques avec le déclin sensible des céréales et des marqueurs pastoraux (pollen et spores de champignons coprophiles) suggèrent une possible rétraction des terres cultivées et une activité pastorale légèrement moins soutenue. Ces changements constatés dans le système d'exploitation peuvent s'expliquer par les troubles importants qui ont lieu dans la région. Les épidémies de peste, dont celle de 1348 qui fait perdre à Tulle une grande partie de sa population ${ }^{44}$, s'ajoutent à la Guerre de Cent Ans qui bat son plein notamment avec la prise de Tulle en 1346 et le sac de Limoges en 1370 ${ }^{45}$. Par ailleurs, les données sédimentologiques révèlent un événement sableux entre 1370 et 1380 cal. p.C. (Unité VI, fig. 7) approximativement signant probablement l'entrée dans le Petit Age Glaciaire dont on sait que les prémices, notamment le XIV ${ }^{\mathrm{e}}$ s., furent particulièrement froids ${ }^{46}$. Différentes sources historiographiques évoquent elles aussi les nombreux aléas climatiques que subit le Limousin durant le XIV s. De nombreuses "Annales", comme les Annales de Saint-Martial, décrivent de très fortes pluies torrentielles gonflant de nombreux cours d'eau et provoquant des inondations ${ }^{47}$. Les parts respectives entre les facteurs naturels - notamment climatiques - et les bouleversements socio-politiques et démographiques ne sont pas faciles à discriminer dans les changements des pratiques territoriales constatés. Les données palynologiques permettent malgré tout de nuancer les sources historiographiques qui parlent de "ruine des campagnes" pour cette période ${ }^{48}$. En effet, aussi importants soient-ils, ces bouleversements ne provoquent pas, dans le secteur considéré, de véritable déprise agro-pastorale ni d'abandon des terres comme c'est le cas dans d'autres massifs de moyenne montagne comme le Jura9 ${ }^{49}$ (zone pollinique M3-b).

Cette tendance au retour des formations forestières régionales s'accentue entre la seconde moitié du XV et le $\mathrm{XVIII}^{\mathrm{e}}$ s. (zone pollinique M-4). Régionalement, si une certaine fréquentation pastorale persiste assurément, le déclin substantiel des spores de champignons coprophiles indique qu'elle est localement de plus faible intensité. La zone humide de Mars semble même être délaissée et se boise progressivement en

43. Corona et al. 2010, 392.

44. Houdart 1999, 40.

45- Leroux 1906, 155-233; Tricard 1996, 16.

46- Messerli et al. 2000, 470.

47. Tricard, 1996, 36.

48. Tricard 1996, 43.

49. Gauthier 2004, 169. 
Pinus (zone pollinique M4-a et début de la zone M4-b). Toutefois, le secteur est loin d'être abandonné et le village de Mars est même mentionné dans les registres paroissiaux de 1656 et $1793^{50}$. Dans les actes civils du $\mathrm{XVIII}^{\mathrm{e}}$ s., il apparaît même plusieurs mentions de propriétaires, fermiers et cultivateurs indiquant qu'une activité agricole se maintient dans le hameau. Ceci s'accorde en tout point avec les données palynologiques qui indiquent même le démarrage de la culture du sarrasin à partir de la seconde moitié du $\mathrm{XV}^{\mathrm{e}} \mathrm{s}$. approximativement.

\section{Histoire du châtaignier}

Des affinités toutes particulières se sont développées au cours des siècles entre les sociétés limousines et le châtaignier aussi bien sur le plan historique et économique que sur un registre plus sentimental voire identitaire. Pourtant son histoire reste encore méconnue et l'analyse palynologique de la séquence de Mars apporte des éclairages inédits.

La question des origines du châtaignier en Limousin

La question est de savoir si le châtaignier est une plante indigène ou non en Limousin. La réponse n'est pas simple dans la mesure où, encore aujourd'hui, les limites de son aire de diffusion naturelle depuis ses zones refuges du dernier maximum glaciaire sont encore floues. Le châtaignier est une espèce thermophile dont les refuges macro-régionaux connus étaient essentiellement situés dans le sud de l'Europe : la région trans-caucasienne, le nord-ouest de l'Anatolie, l'arrière-pays de la côte tyrrhénienne, le sud de l'Italie et la côte cantabrique ${ }^{51}$. Mais la diffusion de cette essence a pu aussi se faire à partir d'îlots plus résiduels localisés dans des zones où un micro-climat a pu assurer leur maintien ${ }^{52}$. C'est très probablement le cas de l'Aquitaine et plus spécifiquement de la Dordogne. Déjà, les découvertes de fragments carbonisés de racines de châtaignier dans des dépôts magdaléniens ${ }^{53}$ ou de charbons de bois dans la grotte de Lascaux ${ }^{54}$ avaient suffi à émettre l'hypothèse de l'indigénat du châtaignier dans le Périgord. Plus récemment, en Dordogne, de nouvelles recherches palynologiques attestent une présence ancienne de châtaignier, à partir de $2000 \mathrm{cal}$. a.C. ${ }^{55}$. À l'inverse des analyses menées dans des sédiments minéraux archéologiques du sud-ouest de la France évoquant l'indigénat du châtaignier - remis en cause par Coûteaux ${ }^{56}$ - aucun problème de pollution ne peut être invoqué dans ces nouvelles analyses réalisées dans des fonds de vallons de la Dordogne, de la Crempse, de la Dronne et de la Nizonne.

L'existence possible de micro-refuges dans un secteur proche du Limousin relance la question du réel statut de cet arbre. À la fin des années 90, M.-F. Diot avance l'hypothèse de l'indigénat du châtaignier en Limousin à partir de l'analyse de niveaux datés du Premier âge du Fer (entre c. 745 et 405 cal. a.C.) provenant de fonds de vallon de la Haute-Vienne (sites du Temple et des Genêts, Vicq-sur-Breuil ${ }^{57}$ ). Ces données venaient contredire l'idée, communément admise jusque là, qu'en Limousin, l'introduction du châtaignier avait été réalisée au rythme de la romanisation des campagnes. En effet, les données anthracologiques avaient toujours évoqué une apparition récente de cet arbre dans la mesure où aucun charbon de bois de châtaignier n'avait été mis au jour dans un site protohistorique et que le plus ancien site a avoir fourni du

50- Références respectives E_DEP85GG-1 et E_DEP85E-1, Archives départementales de la Corrèze, commune de Gimel-les-Cascades.

51. Krebs et al. 2004, 145.

52. Krebs et al. 2004, 145.

53. Fliche 1907, 132-136.

54- Leroi-Gourhan 1979.

55. Leroyer 2010, 217.

56. Coûteaux 1981, 433.

57- Allée et al. 1997, 368-373. 
châtaignier était rapporté à une période s'étalant entre le $\mathrm{I}^{\mathrm{er}}$ et le IV s. p.C. (villa gallo-romaine des Couvents à la Chapelle-Montbrandeix en Haute-Vienne ${ }^{58}$ ).

L'étude de nombreuses séquences tourbeuses de la montagne limousine, particulièrement bien datées, indique une présence assez récente du châtaignier ${ }^{59}$. Elle est dans tous les cas postérieure aux grands défrichements datés entre les $I^{e}{ }^{e}$ et II ${ }^{e} s$. a.C. et à la mise en place des prairies permanentes et d'importantes cultures céréalières ${ }^{60}$, tendant à confirmer le rôle prépondérant de l'homme dans sa diffusion. Les premières occurrences polliniques de châtaignier sont même datées du changement d'ère sur le site de la Font-Carluze (Pérols-sur-Vézère) ${ }^{61}$. En raison de son démarrage plus tardif, entre les $\mathrm{IV}^{\mathrm{e}}$ et $\mathrm{VI}^{\mathrm{e}} \mathrm{s}$. p.C., la séquence de Mars ne permet malheureusement pas d'apporter de nouvelles informations sur les bas-plateaux de Tulle. Or, en raison entre autres de sa plus faible altitude, ce secteur aurait pu constituer un meilleur micro-environnement refuge du châtaignier que le plateau de Millevaches. C'est pourquoi nous présentons également ici la séquence inédite des Chaux-de-Coudert (Clergoux, Corrèze), dont le démarrage sédimentaire est daté de c. 900-520 cal. a.C. (2590 \pm 70 BP VRI-2114). Dès la période du Hallstatt, le milieu a déjà fait l'objet d'éclaircissements forestiers et une fréquentation pastorale est attestée dans les environs du site (zone pollinique $\mathrm{Cl}$-a, fig. 8). Mais c'est surtout à une période, rapportée à la conquête romaine, qu'une vaste opération de déforestation et d'essor des activités agro-pastorales est menée (zone pollinique Cl-c). C'est alors qu'apparaissent les premières occurrences polliniques de châtaignier. L'ensemble de ces analyses polliniques récentes indique une introduction romaine du châtaignier en Corrèze, dès les tous premiers siècles après la conquête. Récemment, sur le site du Cazaudet (Malemort-sur-Corrèze), des châtaignes ont été retrouvées

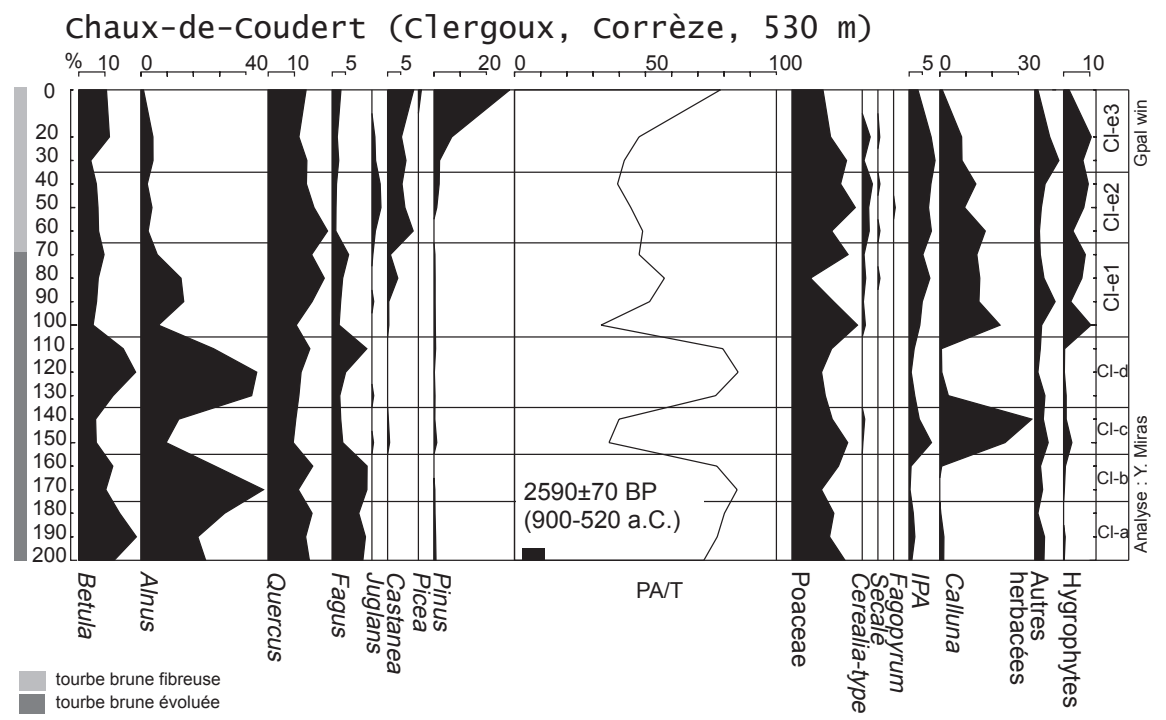

Fig. 8. Extrait du diagramme pollinique en fréquences relatives des Chaux de Coudert.

58- Fredon 1984, 11-13.

59. Guenet 1992, 515

60- Miras et al. 2010, 107.

61- Miras et al. 2010, 122. 
dans un comblement de puits daté du II - -III ${ }^{\mathrm{e}}$ s. p.C. ${ }^{62}$. Les observations de grains de pollen de châtaignier de l'âge du Fer de la Haute-Vienne paraissent donc isolées face au large faisceau d'indices archéobotaniques (palynologique, anthracologique et carpologique) invoquant son introduction romaine. M.-F. Diot évoque "quelques grains de pollen" retrouvés de surcroît dans les tous premiers niveaux organiques surmontant des apports sablo-graveleux ${ }^{63}$, ne permettant donc pas d'exclure totalement des remaniements. De plus, des études récentes démontrent que dans le cas de très faibles fréquences polliniques de Castanea, il est difficile - pour tout palynologue - de trancher définitivement sur l'authentification de ce pollen tant sa morphologie est proche de plantes courantes en Limousin telles Lotus, Hypericum ou Sedum ${ }^{64}$. Il n'est donc pas possible pour l'instant de conclure sur l'indigénat de Castanea en Limousin sur la base de ce seul résultat.

Une place de plus en plus prégnante dans le système d'exploitation

L'étude palynologique des séquences tourbeuses de Mars et des Chaux-de-Coudert convergent sur le fait que si les perceptions polliniques de châtaignier se multiplient durant l'Antiquité, celles-ci restent tout de même assez modérées. Cependant l'observation même faible de grains de pollen de châtaignier est suffisante pour attester sa présence locale ${ }^{65}$. Cet arbre présente en effet une faible production pollinique et des capacités de dissémination réduites étant donné son double mode de pollinisation, à la fois entomophile et anémophile $\mathrm{e}^{66}$. Aussi, entre la fin du IV et le VIII $\mathrm{e}$ s. p.C., une présence discrète de châtaignier est envisageable à Mars (zone pollinique M-1). On peut imaginer des petits bosquets situés à la lisière des chênaies et des chênaies-hêtraies. Cette présence modeste du châtaignier en Limousin semble être confirmée par le nombre assez réduit de sites gallo-romains ayant livré des témoignages botaniques ${ }^{67}$. En Italie, dans l'ancienne Campanie, cette présence modérée du châtaignier dans le paysage correspond à des plantations limitées mais proches des villae occupées entre le $\mathrm{II}^{\mathrm{e}}$ et le $\mathrm{IV}^{\mathrm{e}}$ s. p.C. ${ }^{68}$. Un scénario similaire semble se dessiner pour le Limousin où ces petites populations de châtaigniers, proches des habitats, semblent moins destinées à la culture du fruit qu'à la production d'un bois d'œuvre et d'un bois de chauffe à vocation domestique voire proto-industrielle. En effet, sur le site des Couvents ( $\mathrm{I}^{\mathrm{er}}-\mathrm{IV} \mathrm{v}^{\mathrm{e}} \mathrm{s}$. p.C.), J.J. Fredon ${ }^{69}$ rapporte son utilisation probable pour la fonte de minerai de fer. Sur le plateau de Millevaches, à moins de $200 \mathrm{~m}$ de la zone humide de la Font Carluze, - séquence où des grains de pollen de châtaignier ont été notés dès le I ${ }^{\text {er }}$ s. p.C. ${ }^{70}-$ de nombreux fragments de tegulae et des tessons de céramique marquent l'emplacement d'un habitat galloromain rapporté aux II IIII $^{\mathrm{e}}$ s. p.C..$^{71}$. En effet, l'étude dendrochronologique des canalisations en bois situe la date d'abattage pour le dernier cerne autour de 193 p.C. ${ }^{72}$. D'après G. Lintz ${ }^{73}$, ce système de canalisation n'est pas réservé à un usage particulier et pourrait, entre autres hypothèses, servir à acheminer l'eau vers une installation de lavage du minerai de fer d'autant plus que des scories de fer ont été découvertes à moins de $200 \mathrm{~m}$ au sud-ouest de la partie fouillée ${ }^{74}$. Ceci implique que le châtaignier a dû rapidement se trouver en grande quantité en Limousin car les activités métallurgiques nécessitent beaucoup de bois. Il est donc envisageable que cette essence ait été d'abord cultivée sous la forme de taillis. Des cycles de rotation courts pourraient ainsi expliquer les faibles concentrations polliniques observées pour cette période. Ces similitudes

62. Bouchette com. pers.

63. Allée et al. 1997, 369.

64. Krebs et al. 2004, 147.

65. Conedera et al. 2004, 162-163.

66- Paillet et al. 1991, 440.

67. Fredon 1984, 14.

68- di Pasquale et al. 2010, 869-871.

69. Fredon 1984, 14

70. Miras et al. 2010, 122.

71- Lintz 1977, 43.

72. Lintz 1992, 84.

73. Lintz 1977, 65.

74. Lintz 1977, 44. 
entre le Limousin et la Campanie ${ }^{75}$ permettent de comprendre que les Romains n'introduisent pas tant l'arbre en lui-même mais plutôt un schéma de pensée, une certaine idée de cette plante et de ses utilisations ${ }^{76}$.

À Mars, cette situation semble perdurer jusqu'au Moyen Âge et plus particulièrement jusqu'aux XII ${ }^{\mathrm{e}}$-XIII ${ }^{\mathrm{e}} \mathrm{s}$. qui constituent une première période-clé d'expansion de sa culture (zone pollinique M-2 et Cl-e2, fig. 7 et 8 ). Les plantations de châtaigniers progressent dans les environs de la zone humide. Cette essence est thermophile et elle a pu être également favorisée par la hausse des températures caractéristiques de l'optimum climatique médiéval ${ }^{77}$. Mais le facteur déterminant semble bien anthropique dans la mesure où l'essor de la culture du châtaignier se fait dans un contexte général d'emprise agro-pastorale. Il est intéressant de noter que les toponymes se rapportant au châtaignier se multiplient dans les documents historiographiques. Ainsi, en 930 p.C., la villa Castanedus, de la vicairie de Chamboulive, est cédée par Raingold à l'abbaye de Tulle ${ }^{78}$. Les cartulaires d'Uzerche et de Vigeois, datant du début du XII ${ }^{\mathrm{e}}$ s., mentionnent près d'une trentaine de manses, borderies ou prieurés portant un nom dérivé du châtaignier ${ }^{79}$. Toutefois, d'après l'analyse pollinique, ces plantations ne devaient pas encore recouvrir de vastes étendues dans le secteur de Mars. Aussi, si cet arbre joue un plus grand rôle dans l'économie corrézienne du Moyen Âge, il ne semble pas encore être l'un de ses fondements comme dans le Périgord où il intervient aussi bien dans l'alimentation, l'artisanat que dans les activités proto-industrielles ${ }^{80}$. Ceci s'accorde assez bien avec les données historiques, et notamment les contrats de fermage et de métayage qui ne mentionnent jamais la châtaigne contrairement au seigle ${ }^{81}$.

C'est en fait à partir de la seconde moitié du XIVe s. approximativement que la culture du châtaignier connaitt un important essor (zone pollinique M-3). Sachant que cet arbre est toujours sous-représenté sur le plan pollinique ${ }^{82}$, les fréquences maximales constatées oscillant autour de $20 \%$ attestent la formation de vastes "forêts" de châtaigniers dans le secteur de Mars, probablement les premières châtaigneraies-vergers. Le système agro-sylvo-pastoral se diversifie et laisse une large place à la culture du châtaignier qui en devient rapidement un élément fondamental. Ceci s'explique probablement par ses nombreux avantages : la châtaigne pour l'alimentation quotidienne des hommes et du bétail, les feuilles pour le fourrage et la litière, le bois pour le chauffage ou comme bois d'œuvre. Il semble que ce soit principalement au détriment des terres cultivées, toujours présentes mais moins étendues que durant le Moyen Âge classique, que se développent les plantations de châtaigniers. Cette situation perdure jusqu'au milieu du $\mathrm{XV}^{\mathrm{e}} \mathrm{s}$. Cette production en Limousin semble, à ces périodes, surtout se fonder sur une économie d'auto-subsistance, une culture à l'échelle du hameau et de la ferme limousine ${ }^{83}$. Postérieurement, une inflexion notable dans les fréquences polliniques, dont les taux moyens ne sont plus que de $10 \%$, laisse envisager soit une rétraction des plantations soit un changement de gestion impliquant un nouveau faciès des plantations (zone pollinique M-4a). Un nouvel essor de la châtaigneraie-taillis pourrait servir les intérêts d'activités métallurgiques. Cette activité est très coûteuse en bois et semble être un moteur de l'activité de charbonnage ${ }^{84}$. Dans le cadre du Programme Collectif de Recherche "Géohistoire et géoarchéologie des territoires forestiers limousins" (dir. P. Allée), les travaux de R. Rouaud (thèse en cours) montrent que le charbonnage est pratiqué depuis le $\mathrm{XV}^{\mathrm{e}} \mathrm{s}$. avec un

75- di Pasquale et al. 2010, 871 .

76. Conedera et al. 2004, 176.

77. Paillet et al. 1991, 440.

78- Fage 1917, 142.

79. Pouget 2002, 19.

80- Lacombe 2007, 49 .

81. Pouget 2002, 19.

82- Paillet et al. 1991, 432.

83. Plas \& Boumédiene 2010, 168.

84- Allée 2007-2009. 
maximum d'activité entre 1750 et $1900^{85}$. Dans le diagramme pollinique de Mars (zone pollinique M-4b), à cette période, les pourcentages de châtaignier régressent de nouveau signant très probablement la rétraction des châtaigneraies-vergers au profit d'un essor de sa culture sous la forme de taillis. Les prospections réalisées en Corrèze par R. Rouaud le long de la haute vallée de la Dordogne jusqu'à Argentat et le long de la vallée de la Luzège montrent une très grande densité de plateformes de charbonnage ${ }^{86}$ (fig. 1). D'autres facteurs expliquent aussi ce recul des châtaigneraies fruitières comme l'introduction de la pomme de terre, qui, encouragée par la politique de Turgot, supplante peu à peu - quoique progressivement et non sans difficultés - la châtaigne ${ }^{87}$. Les progrès généralisés des techniques agricoles tout comme l'essor de la microindustrie du feuillard du début du XX $\mathrm{Xx}^{\mathrm{e}} \mathrm{s}$. nécessitant du bois de taillis ${ }^{88}$ contribuent aussi à cette situation. Les plantations résiduelles de châtaigniers de vergers servent alors pour l'obtention de bois de chauffe ou de construction ou bien pour les industries tannantes ${ }^{89}$. Malheureusement, la séquence de Mars, en raison de la qualité de son enregistrement, ne permet pas de détailler finement les vicissitudes de la culture du châtaignier en Corrèze pour les temps postérieurs au début de la Renaissance.

\section{Un marqueur paysager aux connotations contradictoires et fluctuantes}

Les liens tissés au cours du temps entre le châtaignier et les sociétés limousines sont tellement anciens et entremêlés que cette plante est devenue un véritable marqueur paysager et territoria ${ }^{90}$. Mais sa perception est variable au fil des siècles et l'analyse palynologique de la séquence de Mars permet d'analyser l'histoire de ces relations sous un prisme inédit.

Les sociétés de l'Antiquité ne sont certes pas indifférentes à l'utilité du châtaignier, mais les rapports qu'elles entretiennent avec cet arbre semblent empreints d'une certaine neutralité. Il est en effet considéré comme un arbre comme un autre au même titre que le hêtre ou le chêne. Essentiellement utilisé comme bois combustible et bois d'œuvre, son usage dans l'alimentation ne paraît pas être la priorité. Les sociétés antiques ne passent pas pour autant "à côté" de cet arbre et on peut tout de même imaginer des cultures d'appoint. La châtaigne est malgré tout consommée à Rome. Les écrits de Pline l'Ancien détaillent les méthodes de sa culture et donnent quelques recettes pour cuisiner ce fruit ${ }^{91}$. Toutefois, les Romains - et particulièrement la classe dominante - ne semblent guère apprécier la châtaigne à l'instar de l'Amaryllis de Virgile comme le rappelle J.R. Pitte ${ }^{92}$. Le plus frappant est de voir qu'on retrouve cette perception somme toute assez négative durant le $\mathrm{XIX}^{\mathrm{e}} \mathrm{s}$. où l'on considère comme archaïque de cultiver le châtaignier ${ }^{93}$. Les agronomes du XIX ${ }^{e}$ s. ramenèrent la culture de la châtaigne à une simple cueillette, incitant à la paresse. Le châtaignier est même catalogué comme un marqueur primitif antérieur à tout processus de civilisation. Pourtant, les données palynologiques récemment obtenues dans le Pays de Tulle ou sur la Montagne limousine démontrent très clairement le contraire. La culture du châtaignier est en Limousin postérieure de près de cinq millénaires aux premières activités agro-pastorales du Néolithique ancien ${ }^{94}$ ! Elle est l'expression de cultures ayant développé des systèmes d'exploitation diversifiés et de pratiques territoriales évolutives. Ces phases de perception négative coïncident le plus souvent avec une utilisation du châtaigner principalement orientée vers le bois d'œuvre et de chauffage voire pour des utilisations industrielles (industrie tannante par

85. Rouaud 2011, 84.

86- Allée 20072009.

87. Houdart 1999, 57.

88. Rouaud et al. sous presse, 4

89. Rouaud et al. sous presse, 4.

90- Grandcoing 2010, 275.

91- Conedera et al. 2004, 172.

92. Pitte 1986, 60.

93. Grandcoing 2010, 277

94- Miras et al. 2011, 158. 
exemple ${ }^{95}$ ). C'est dans ce sens qu'E. Lamy de Lachapelle, en 1839, conseille la culture du châtaignier dans ses articles scientifiques ${ }^{96}$. La châtaigne, comme pour la période antique, n'est pas ou plus à la base de l'alimentation ou pire, elle est vue comme un "mal nécessaire" en période de disette ou d'exode rura197. C'est donc plus que de simples utilisations d'une essence végétale dont il est question ici, mais de véritables projections d'une société sur celles-ci.

Cette conception s'oppose aux sociétés rurales de la fin du Moyen Âge et des Temps modernes souvent qualifiées de "civilisations de la châtaigne". Au XVIII" s., on parle même de "l'arbre à pain" tant la châtaigne est importante dans le régime alimentaire des familles françaises ${ }^{98}$. Contrairement à l'Antiquité, même l'aristocratie de l'Ancien Régime n'est pas indifférente à ses charmes. Ce rôle de "plante nourricière" est bien sûr à la base de cette valorisation extrême du châtaignier car on touche à ce qui il y a de plus important : se nourrir pour survivre. Une relation plus incarnée et plus vivante se met en place et démarre alors une analogie étroite entre les hommes et le châtaignier. Celle-ci trouvera, particulièrement à la fin du XIX ${ }^{e}$ s., un nouveau catalyseur à l'origine d'une nouvelle forme de valorisation symbolique du châtaignier : la mémoire historique. Le châtaignier est vu comme le témoin ancestral de l'histoire humaine et ses louanges sont chantées dans ce sens ${ }^{99}$. La palynologie permet de saisir toute l'ironie de cette situation dans la mesure où l'on sait maintenant que son introduction est somme toute assez récente et en tout cas postérieure à d'autres végétaux très couramment cultivés en Limousin comme le blé ou le seigle et pour lesquels moins de passions ont été déchaînées. C'est donc avant tout des valeurs culturelles que traduit l'étude de cet arbre y compris aujourd'hui. En Limousin, le châtaignier, véritable outil de communication, est même devenu un emblème identitaire. La feuille de châtaignier est en effet le logo du Conseil Régional du Limousin depuis 1989 et son identité visuelle a été modernisée en 2007. Dans le document explicatif du Conseil Régional Limousin et du Centre de Recherches Sémiotiques de l'université de Limoges (I. Klock-Fontanille) sur la mise en place de ce logo, on apprend que le traitement de la feuille de châtaignier symbolise à la fois "la notion de milieu naturel, de renvoi à la tradition et à la campagne limousine" et, dans sa nouvelle version, d'ouverture, de dynamisme et de force mais aussi de continuité. L'arbre est donc davantage décrit comme une façon qu'a une société de le percevoir plutôt qu'en fonction d'une vérité scientifique. En effet, à la lumière des données paléoenvironnementales et historiques, nous savons que le châtaignier est un arbre importé et cultivé depuis relativement peu de temps à l'échelle de l'histoire française. Il est donc davantage un marqueur anthropique que naturel. Par ailleurs, dans un passé récent, il était considéré comme un stéréotype d'archaïsme et de paresse bien loin de la vision actuelle de modernité et de dynamisme. Les jeux de va-et-vient dans la perception de cette essence durent donc depuis deux millénaires !

\section{CONCLUSION}

Les analyses palynologiques des deux séquences tourbeuses corréziennes de Mars (Gimel-les-Cascades) et des Chaux-de-Coudert (Clergoux) ont permis d'apporter les premières informations d'ordre paléoenvironnemental (palynologie et sédimentologie) dans le Pays de Tulle. Elles démontrent l'intérêt de mener de telles recherches dans la caractérisation de l'exploitation du milieu et de la gestion des ressources végétales sur la longue durée comme l'aménagement des zones humides ou la place de la culture du châtaignier. L'enregistrement sédimentaire exceptionnel de Mars a permis de détailler de façon inédite l'histoire de cette essence depuis son introduction au début de l'Antiquité jusqu'à la Renaissance. Les

95- Rouaud et al. sous presse, 4.

96- Grandcoing 2010, 281.

97. Combet 2007, 87; Grandcoing 2010, 280.

98. Pitte 1986, 112

99. Grandcoing 2010, 284. 
données présentées révèlent que sa culture n'est pas linéaire au cours du temps et que son intégration au sein du système d'exploitation est variable, essentiellement en fonction de facteurs culturels et économiques voire symboliques. C'est la perception ou autrement dit la mentalisation de cette essence par les sociétés de chaque époque qui joue un rôle primordial. Mais les résultats présentés ici ne sont que les premières pierres apportées à l'édifice et de nouvelles analyses paléoenvironnementales, combinées aux données archéohistoriques, devront dans le futur préciser les trajectoires paléopaysagères et les modèles de gestion des ressources naturelles dans les bas-plateaux limousins, encore sous-explorés aujourd'hui.

Allée, P. (2003) : Dynamiques hydrosédimentaires actuelles et holocènes dans les systèmes fluviaux de moyenne montagne (Limousin, Hautes Cévennes, Maures), mémoire d'Habilitation à Diriger des Recherches, université de Limoges.

Allée, P., éd. (2007-2009) : Géohistoire et géoarchéologie des territoires forestiers limousins $(P C R)$, Bilans scientifiques 20072009, DRAC-SRA Limousin, opérations interdépartementales.

Allée, P., M.-F. Diot, J.-F. Durieux, J.-M. Reynet et B. Valadas (1997) : "Trois mille ans d'enregistrement sédimentaire dans les fonds de vallons des bas plateaux limousins. La dynamique des paysages protohistoriques, antiques, médiévaux et modernes", in : Actes des XVIIle Rencontres Internationales d'Archéologie et d'Histoire d'Antibes, Sophia-Antipolis, 365-387.

Barrière, B. (2006) : Limousin médiéval, le temps des créations. Recueil d'articles, Limoges.

Berglund, B.E. (2011) : "How mankind has shaped European nature?", Vegetation History and Archaeobotany, 20, 79-81.

Combet, M. (2007) : "Châtaigne, châtaigneraie en Périgord au XVIII siècle", in : La Châtaigne en Périgord, fruit des Temps et des Hommes, La Lauze Numéro spécial, 87-115.

Conedera, M., P. Krebs, W. Tinner, M. Pradella et D. Torriani (2004) : "The cultivation of Castanea sativa (Mill.) in Europe, from its origin to its diffusion on a continental scale", Vegetation History and Archaeobotany, 13, 161-179.

Corona, C., J. Guiot, J.-L. Edouard, F. Chalié, U. Büntgen, P. Nola et C. Urbinati (2010): "Millenium-long summer temperature variations in the European Alps as reconstructed from tree rings", Climate of the Past, 6, 379-400.

Coûteaux, M. (1981) : "À propos de la signification pollinique de Castanea en Dordogne. Sa dispersion actuelle et sa signification dans les sédiments", Pollen et spores, 23, 3-4, 433-439.

Cubizolle, H., F. Fassion, J. Argant, C. Latour-Argant, P. Galet et C. Oberlin (2011): "Mire initiation, climatic change and agricultural expansion over the course of the Late-Holocene in the Massif Central mountain range (France): Causal links and implications for mire conservation", Quaternary International, 251, 77-96.

Dehlon, C., I. Théry-Parisot et S. Thiébaut, éd. (2010) : Des Hommes et des Plantes, Exploitation du milieu et gestion des ressources végétales de la Préhistoire à nos jours, XXXe Rencontres internationales d'Archéologie et d'Histoire d'Antibes, Antibes.

Denèfle, M., B. Valadas, A. Vilks et G. Lintz (1980) : "L'évolution holocène de la végétation en Limousin", Bulletin de l'Association française de l'Étude du Quaternaire, 4, 189-198.

Desbordes, J.-M. et B. Valadas (1979) : L'érosion agricole en milieu cristallin : processus, conséquence et dimension historique du phénomène à partir d'exemples pris dans le Massif d'Ambazac (Limousin), Actes du colloque sur l'érosion agricole des sols en milieu tempéré non méditerranéen, Strasbourg, 19-25.

Diot, M.-F. (1987) : "Palynologie et archéologie : état des recherches en Limousin", TAL, 7, 21-27.

Diot, M.-F. et J.A. Lopez Saez (1998): "Remarques paléoécologiques à propos de quelques palynomorphes non-polliniques provenant de sédiments quaternaires en France", Revue de Paléobiologie, Genève, 17, 445-459.

di Pasquale, G., E. Allevato, E. Russo Ermolli, S. Coubray, C. Lubritto, F. Marzaioli, M. Yoneda, K. Takeuch, Y. Kano, S. Matsuyama et G.-F. de Simone (2010) : "Reworking the idea of chestnut (Castanea sativa Mill.) cultivation in Roman times: new data from ancient Campania", Plant Biosystems, 144, 4, 865-873.

Ejarque, A., Y. Miras et S. Riera Mora (2011): "Modern pollen and non-pollen palynomorph analogues of vegetation and highland grazing activities obtained from surface and dung datasets from the eastern Pyrenees", Review of Palaeobotany and Palynology, 167, 123-139.

Emberger, A. et J.-J. Périchaud (1979) : Carte des ressources minérales du Massif central, Paris.

Faegri, K. et J. Iversen (1989) : Textbook of pollen analysis, $4^{\text {th }}$ ed., New Jersey.

Fage, R. (1917) : La propriété rurale en Bas-Limousin pendant le Moyen Age, Paris.

Fau, L., éd. (1996) : Les Monts d'Aubrac au Moyen Age. Genèse d'un monde agro-pastoral, DAF 101, Paris.

Fliche, P. (1907) : "Note sur un charbon quaternaire de châtaignier", Bulletin de la Société Botanique de France, 54, 132-136.

Fredon, J.-J. (1984) : "Macrorestes végétaux de sites archéologiques en Limousin", TAL, 5, 7-15. 
Gauthier, E. (2004) : Forêts et agriculteurs du Jura. Les quatre derniers millénaires, Besançon.

Galop, D. et C. Servelle (2006) : "Présentation géographique et géomorphologique L'anthropisation du plateau de l'Aubrac à partir des données polliniques", in : Fau, éd. 1996, 18-36.

Goeury, C. (1997) : GPa/Win: gestion, traitement et représentation des données de la paléoécologie, Actes du XV' symposium de I'APLF, Lyon, 31.

Grandcoing, P. (2010) : "La naissance d'un marqueur paysager régional : le Limousin et le châtaignier", in : Grandcoing, éd. 2010, 275-293.

Grandcoing, P., éd. (2010) : Paysage et environnement en Limousin, de l'Antiquité à nos jours, Limoges.

- (sous presse) : Industrie et monde rural en France de l'Antiquité au xxe siècle, Limoges.

Guenet, P. (1992) : L'impact de l'homme sur son environnement en moyenne montagne, du Cézallier au plateau de Millevaches (Massif central, France), Actes du $117^{\mathrm{e}}$ Congrès national des Sociétés savantes, Clermont-Ferrand, 515-528.

Haas, J.-N. (1996) : "Neorhabdocoela oocytes - palaeoecological indicators found in pollen preparations from Holocene freshwater lake sediments", Review of Palaeobotany and Palynology, 91, 371-382.

Houdart, M.-F. (1999) : Pays et paysans du Limousin, Tulle.

Jacobson, G.L. et R.H.W. Bradshaw (1981) : "The selection of sites for palaeovegetational studies", Quaternary research, 16, 8096.

Jouffroy-Bapicot, I. (2010) : Évolution de la végétation du massif du Morvan (Bourgogne - France) depuis la dernière glaciation à partir de l'analyse pollinique. Variations climatiques et impact des activités anthropiques, Thèse, université de Franche-Comté.

Krebs, P., M. Conedera, M. Pradella, D. Torriani, M. Felber et W. Tinner (2004): "Quaternary refugia of the sweet chestnut (Castanea sativa Mill.): an extended palynological approach", Vegetation History and Archaeobotany, 13, 145-160.

Lacombe, C. (2007) : "Des châtaigniers et des châtaignes en Périgord entre le $x^{e}$ et le $x v \|^{e}$ siècles", in : La Châtaigne en Périgord, fruit des Temps et des Hommes, Périgueux, numéro spécial, La Lauze, 49-84.

Lavrieux, M., J. Jacob, J.R. Disnar, J.G. Bréheret, C. Le Milbeau, Y. Miras et V. Andrieu-Ponel (2013): "Sedimentary cannabinol tracks the history of hemp retting", Geology, 41, 751-754.

Leroi-Gourhan, A., L. Balout, C. Bassier, R. Bouchez, J. Bouchud et B. Couraud (1979) : Lascaux inconnue, Paris.

Leroux, A. (1906) : "Le sac de la cité de Limoges et son relèvement, 1370-1464", Bulletin de la Société archéologique et historique du Limousin, 56, 155-233.

Leroyer, C. (2010) : Apparition et diffusion du châtaignier (Castanea sativa) en Dordogne : I'apport de la palynologie, in : Dehlon et al., éd. 2010, 211-224.

Lintz, G. (1977) : "Les canalisations gallo-romaines en bois", Bulletin de la Société des Lettres, Sciences et Arts de la Corrèze, 80, 43-66.

— (1992) : La Corrèze, CAG 19, Paris.

Maniquet, C. (2008) : "Le dépôt cultuel du sanctuaire gaulois de Tintignac à Naves (Corrèze)", Gallia, 65, 19, 273-326.

Messerli, B., M. Grosjean, T. Hofer, L. Nùñez et C. Pfister (2000) : "From nature-dominated to human-dominated environmental changes", Quaternary Science Reviews, 19, 459-479.
Miras, Y. (2009) : "L'étude des relations entre végétation et pluie pollinique actuelle sur le plateau de Millevaches (Limousin, France) : outil pour une meilleure caractérisation pollenanalytique des formes paysagères et des pratiques agrosylvo-pastorales", Revue des Sciences Naturelles d'Auvergne, 73, 71-105.

Miras, Y, P. Guenet et H. Richard (2010) : "La genèse du paysage culturel du Plateau de Millevaches (Limousin, Massif central, France) : plus de 2000 ans d'histoire révélés par l'analyse pollinique", in : Grandcoing 2010, 99-124.

- (2011) : "Holocene vegetation, landscape and reconstruction of human activity from Prehistory to Roman times based on new pollen data performed in the "plateau de Millevaches" (Limousin, Massif Central, France)", Quaternaire, 22, 2, 147164.

Paillet, F.L., M.G. Winkler et P.R. Sanford (1991) : "Relationship between polster and forest composition in a naturalized stand of American chestnut: implication for paleoenvironmental interpretation", Bulletin of the Torrey Botanical Club, 118, 4, 432-443.

Pitte, J.-R. (1986) : Terres de Castanide, Paris.

Plas, P. et F. Boumediene (2010) : "Les évolutions de la châtaigneraie en Limousin-Périgord de la fin du xvII au milieu du xxe siècle", in : Grandcoing 2010, 165-199.

Pouget, R. (2002) : Les châtaigniers du Limousin, Lemouzi.

Prat, B. (2006) : Systèmes agro-pastoraux et milieux péri-urbains en Basse Auvergne au cours des trois derniers millénaires : contribution de l'analyse palynologique à l'étude des interactions sociétés-milieux. Thèse, université ClermontFerrand II.

Pulido Avil, M. (2006) : Conséquences de l'anthropisation sur la dynamique post-glaciaire de la végétation dans le sud du Massif Central, France, Thèse, université Aix-Marseille.

Reille, M. (1995-1999) : Pollen et spores d'Europe et d'Afrique du Nord. Atlas photographique, CNRS éditions, Marseille.

Rouaud, R. (2011) : "Impact du charbonnage sur le paysage forestier de la haute vallée de la Dordogne", Cahier d'Études du Groupe d'Histoire des Forêts françaises, 21, 76-88.

Rouaud, R., P. Plas, P. Allée, I. Eubas-Rementeria et S. ParadisGrenouillet (sous presse) : "Une autre histoire du châtaignier en Limousin", in : Grandcoing, éd. sous presse, Limoges.

Serra, O. (1979) : Acquisition des données diagraphiques, in Diagraphies différées - bases de l'interprétation, Bulletin des Centres de Recherches exploration-Production Elf-Aquitaine Pau.

Serra, O. (1985) : Interprétation des données diagraphiques, in Diagraphies différées - bases de l'interprétation, Bulletin des Centres de Recherches exploration-Production Elf-Aquitaine Pau.

Stuiver, M. et J.P. Reimer (2005) : Radiocarbon Calibration Program Calib. Rev. 5.0.1., http:// calib.qub.ac.uk/calib/calib. html.

Toledo i Mur, A., M. Vacca-Goutoulli et F. Milor (2005) : "Une mine d'or protohistorique : le Puy-des-Angles à Angles-sur-Corrèze", Gallia, 62, 171-214

Tricard, J. (1996) : Les campagnes limousines du XIV au XVe siècles. Originalité et limites d'une reconstruction rurale, Paris.

van Geel, B. (1978) : "A palaeoecological study of Holocene peat bog sections in Germany and the Netherlands", Review of Palaeobotany and Palynology, 25, 1-120.

Vazeille, M. (1962) : La très vieille histoire locale. Le pays d'Ussel, Tulle. 